Published in proceedings of 2019 IEEE 7th International Conference on Serious Games and Applications for Health (SeGAH), Kyoto, Japan, 5-7 august, which should be cited to refer to this work.

DOI: https://doi.org/10.1109/SeGAH.2019.8882466

(C) 2019 IEEE. Personal use of this material is permitted. Permission from IEEE must be obtained for all other uses, in any current or future media, including reprinting/republishing this material for advertising or promotional purposes, creating new collective works, for resale or redistribution to servers or lists, or reuse of any copyrighted component of this work in other works.

\title{
A Picture-based Serious Game to Train Non-medical People for Emergency Situations
}

\author{
Stephane Gobron \\ Engineering School of HE-Arc \\ University of Applied Sciences Western Switzerland (HES-SO) \\ Neuchatel, Switzerland \\ stephane.gobron@he-arc.ch
}

\author{
Sylvie Chatelain \\ Grangette Early Childhood Institute \\ Lausanne Children Day-care Reception Service \\ Lausanne, Switzerland \\ sylvie.chatelain@lausanne.ch
}

\author{
Christophe Bolinhas \\ Engineering School of HE-Arc \\ HES-SO \\ christophebolinhas@gmail.com
}

\author{
Domingos Correia de Oliveira \\ Health School of the canton of Vaud (HESAV) \\ HES-SO
}

Lausanne, Switzerland

dominique.correiadeoliveira@hesav.ch

\begin{abstract}
As a non-medical professional, reacting to an emergency situation where life is at stake is not an easy task: knowing the minimum procedure to react to such a situation is a question that most people just cannot answer. This paper proposes a serious game using a simple picture-based navigation which provides a training tool for common people. This serious game wants above all that everyone can fully know the four priorities of action: (1) keep calm, (2) evaluate the situation, (3) identify the dangers, and (4), call for help.

For practical purposes, the domain of early childhood has been selected to train educators for five $t$ ypical $m$ ajor a ccidents they will probably encounter with children. Two of these accidents were fully developed, parameterized, and tested. This paper focuses are: first, on the medical and social needs, demonstrating how important first-witness reactions are in an emergency scene; second, on how different domain experts and professionals were brought together; third, on how the software was developed based on an educator-centered design. Result in terms of image-based interface and user tests show that a application without fantastic graphics and special effects can have a real impact: potentially, an impact on people's lives.
\end{abstract}

Index Terms-Serious game (SG), Picture-based application, Emergency situation, Childhood, Kindergarten, Educator

\section{INTRODUCTION}

This paper presents how a low-cost photo-based serious game (SG) can help non-medical experts respond to emergency situations. As illustrated in Fig. 1, this paper concerns three domains: (1) healthcare, for the medical advice and official s ystematic $\mathrm{p}$ rocedures $\mathrm{g}$ iven $\mathrm{b}$ y $\mathrm{t}$ he $\mathrm{c}$ entral $\mathrm{c}$ all service; (2) education, as the application domain involves large groups of young children educators; and (3), engineering, for the software application development and game technology advice. The following sections introduce each domain context: Healthcare, Social, and Engineering.

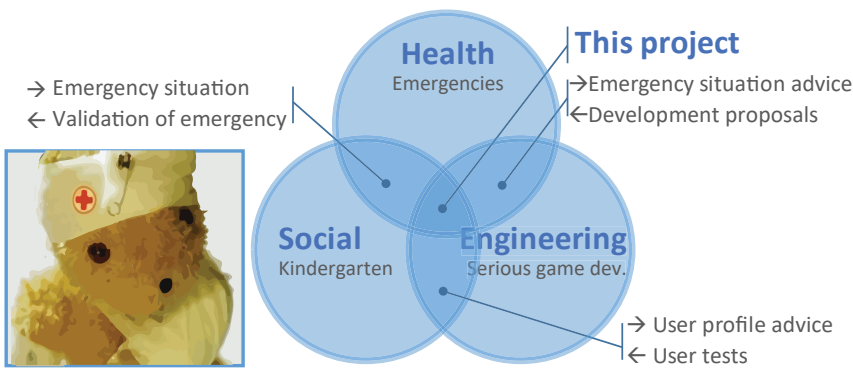

Fig. 1. General presentation of the three domains involved to develop a functional serious game (SG) application to train young child educators for emergency situations.

\section{A. Healthcare context}

Everyday life accidents during the early childhood are a major public health problem as they are the leading cause of accidental infant mortality [11], [12]. Professionals in charge of children often find it difficult to estimate the accident risks related to the child age because they often misunderstand the prevention technical means. In 2013, according to the American National Center for Injury Prevention and Control, an estimated 8.3 million children, aged 19 and under, were seen in emergency departments for non-fatal unintentional injuries, with an injury rate of 10.0 per 100 children, and seven thousand children, aged 19 and under, died from unintentional injuries, the death rate being 9.3 per 100,000 children [22].

Considering the recommendations for collective children day care in Vaud canton, Switzerland, the knowledge and implementation of emergency procedures should be ensured by a continuous training of the employees. The management of each nursery should organize specific training sessions to manage first-aid situations in order to ensure an adequate knowledge level. We thus wanted to understand if the use of a technological tool like a SG would ease the learning processes 
of first-aid management. Indeed, it is possible and necessary to do more and better and go further in the early childhood worker training, by regular recycling and strengthening of their skills in terms of preparation and response to save lives.

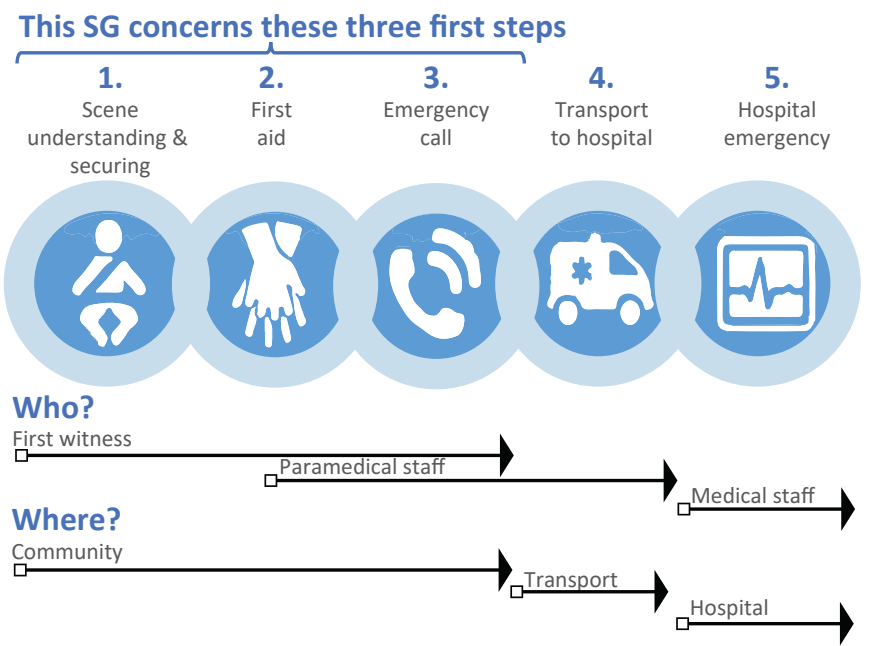

Fig. 2. Pediatric Chain of Survival 2010 - AHA

Public health issues and context - How to increase the survival chances during a life emergency? Different studies such as [24] [15] suggest that the first links in the survival chain (illustrated in Fig. 2) who have the mission to alert the emergency services as quickly as possible, taking care to specify the victim status, must be improved. These first actions will have to be carried out by first witnesses implying how important continuous training of any individual, collaborator or professional can be. Indeed, responding correctly to an emergency situation is difficult since knowledge related to the field is not enough: scene observation skill, initiative skills, and being able to give relevant and accurate information to the "144" (emergency number in Switzerland) are needed and we believe, can be trained to anyone. Immediate initiation of cardiopulmonary resuscitation from witnesses is the main avenue to improve the survival rate after a cardiopulmonary arrest (CPR). The higher the proportion of ACR patients benefiting from first-witness heart massage, the better the overall survival will be with a quality neurological outcome [20].

Action - Concerning the fact people must or have to assist, it differs for each country; regarding Switzerland, Article 128 of the Criminal Code states that non-assistance to persons in danger is punishable by law. We expect each stakeholder to respond to the seriousness of the situation based on their skills and level of responsibility, but at a minimum. Each witness should be able to identify the situation and mobilize relief appropriately through the call to the emergency number and the transmission of targeted information hence the relevance of the game and its added value.
Target - In addition to the efforts made to prevent accidents and diseases, and also progress made in public health, the needs of specific populations must be taken into account, especially those of early childhood workers facing daily first aid. The rescue chain partners (e.g. 144, Police, or REGA -the Swiss Air Guard Rescue) are also working on this weakness by trying to build tools to better understand their interlocutors. Our approach is not to supplant current effort, it is rather complementary. Indeed, we propose to increase the reaction and communication skills of these interlocutors by integrating such serious game for instance at health care bachelor level.

Objectives - An extension could also be developed specifically for parents, as part of prevention courses set up by childcare centres, for example. However, the emotional dimension should be adapted: we would have an increased risk of generating anxiety among participating parents who could project their own child, injured or worse, in the proposed situations. Through the development of this prototype, a pedagogical objective was also to validate the use of this technology as a complementary strategy to the classical training on first aid.

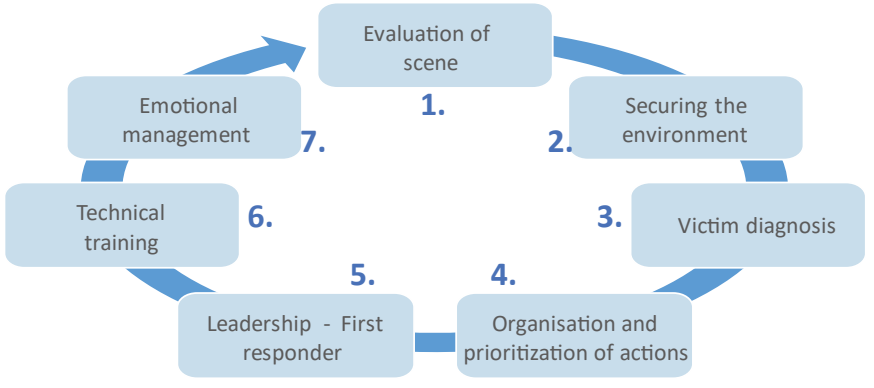

Fig. 3. Different stages targeted by the training with this SG.

Based on the steps illustrated in Fig. 3, our priority objectives to enhance the efficiency of these first links are: skill development; observation; initiative taking; emotion management; decision-making capacity; organization; transmission of relevant information to the emergency controller (911 in the US, 112 in EU, or 144 in Switzerland).

In the longer term, this project will enable to validate the vital-emergency-management basic knowledge and integrate this project as a new pedagogical approach into current acute care training plans. We can imagine the hypothesis that this game can immerse each user in a realistic scenario. Therefore, one of the major goals of the game is to realize its needs to improve dimensions such as leadership, delegation, prioritization of decisions, management of the unexpected.

\section{B. Social context}

In their daily works, professionals supervising young children in community must deal with several missions: educational, social, political and preventive. In this last part, the main task is first and foremost to guarantee the safety of the children in attendance. Extended-time facilities are open 
between 10 and 12 hours a day. Most of them attend to children from the end of their mothers' maternity leave four months - to their schooling or even to the end of their second year at school. Within a day, children live in a group of indoor and outdoor activities responding to their great need for discovery, experimentation and learning. Furthermore, meals, naps, moments of care are added to playful periods.

Despite the constant concern of professionals to ensure the children safety, there are numerous situations likely to cause accidents. Fortunately, the majority of injuries are benign; nevertheless, few cases require the intervention of emergency staff, e.g. deep wounds, broken limbs, unexplained fainting, convulsions. The Swiss Health Observatory Institute ([OBSAN2017]) has identified that children represent around 7\% of the total activity at emergency. We must also add all the socalled special and therefore more sensitive reception situations such as children with disabilities, allergies or epilepsy.

In his professional career, almost any educator will need to call emergencies at least once. Are educators sufficiently prepared? Do they know the essential information that must be transmitted? Are they ready to act despite the strong emotions that a child in distress may arouse? Unfortunately, this is not the case for the large majority. Indeed, in Switzerland, at nursery level, professionals must certify a single day of first-aid training, but nothing is required of teachers. Real skills relative to emergency situations is not popular since, to be valid, it must be refreshed at least once a year, implying heavy costs in time and money. Furthermore, many professionals mistakenly believe that first-aid measures can easily be controlled or that a serious accident just never occurs.

The current SG has strong advantages, in answering these critical issues, at least for educational professionals and we believe it could be easily exported to any institute with a similar structure:

- Accessible at all times to professionals within the institution. It allows a regular knowledge refreshing in a reassuring context;

- The scenario which, without trivializing the accidents, allows the familiarization of the right acts to pose. The professionals are therefore better prepared to act relevantly, as effectively as possible, in the event of an accident;

- The results, experiences or emotions experienced through the SG can lead to team reflections and better targeting of sensitive points to work: emotion management, pain or death in children, collaboration with parents or children management in an accident situation, etc.

Furthermore, based on the impact demonstrated in our institute, it has been demonstrated that such SG is a valuable tool for the whole profession as it promiotes and supports: (1) the information transmission to emergency personnel in case of an accident and makes the injured care more efficient, (2) the services offered and the professional posture by anticipating and knowing the acts to be done in an emergency context, and (3) the managerial work by pointing out essential themes to be explored in an individual and/or collective accompaniment.

\section{Engineering: the serious game states}

Game-based learning is vast and a significant amount of research had been done on its efficiency. Babu et al. [1] demonstrated how a 3D simulation from a collaborative game teaching how to manage post-disaster situations helped the participants to better connect theory to practice and showed the cost efficiency it can bring. In the case of incident triage training, Knight et al. [7] evaluated the effectiveness of a SG that created affordable and accessible interactive virtual worlds. They showed that such an application enhances participants learning and performance in comparison to traditional educational methods. Virtual reality has been explored in pedagogical SG for health for instance with Sisto et al. [19] or Gobron et al. [6] respectively for an approach dealing with musculoskeletal disorder and training for the rehabilitation of the legs involving a head-mounted display and haptic robot devices. Chan et al. researched how a game teaching needle placement [3] could greatly increase the learning of inexperienced medical participants.

As analyzed by the team at the Institute of Digital Media and Child Development Working Group (Baranowki et al. [2]), SGs for health often require less funding because they don't need to compete with AAA entertainment video games budgets. Nowadays research and development for such products are largely funded by government or foundation grants. A study (Li et al. [8]) done in manufacturing environment show evidence that SGs are and effective method to train procedural knowledge in complex manufacturing scenario. Health care procedures are as such procedural knowledge and can become complex as well. Another study (Dieh et al. [5]) proposes the design and development of games for specific handling of chronic diseases treatments, for instance, based on insulin therapy. It shows some potential in developing different game levels for child care institutes. Notice that levels can be easily edit directly through an XML file with a dedicated format. Currently no localization system has been implemented but the current structure would only require and extraction of the current texts into a spreadsheet and the integration of an existing localization module. Furthermore, game data would need to be stored on the cloud to facilitate levels based on various countries legislation.

Many SGs in health or education have been proposed. More specifically for children, we can cite for instance Matsunaga et al. [9] focusing on the development process of an educational game aiming on hemophilia or Mikuszewski et al. [10] with a serious game with therapeutic goals that focuses on issues and problems of preschool children.

\section{DeVelopment STRATEGY}

The work has been developed for and with social educators, paramedical staff, and game designers. The Fig. 4 introduces six categories of actor having participated in this project and representing a total of more that 110 people. Here follows the procedure to bring them together: 


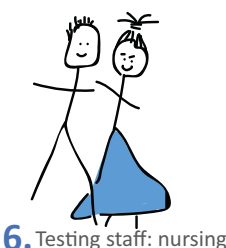

bachelor students -- 60 people

SG tested by 35 people

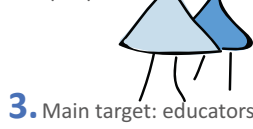

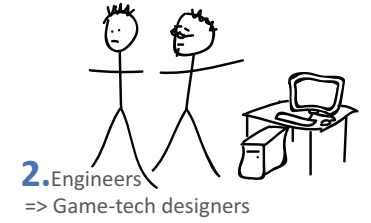

=> Game-tech designers

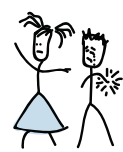

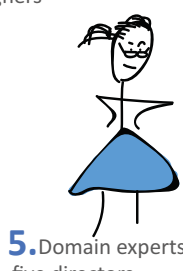

five directors
Fig. 4. This figure presents the six categories of people for a total of 113 people involved in this project.

- Emergency experts (1.) met computer science engineers (2.) to look for appropriate solutions during emergency situations for none medical people;

- Both groups decided to contact educators (3.) as potential application field;

- Educational staff (3.) confirmed their strong needs since the children (4.) they were taking care of often had accidents;

- A mixed group of three software developers and five children institute directors identified ten critical situations, based on their personal experience;

- Five scenarios were designed as a storyboard and two were implemented by (2.): severe double fingers sectioned and premature infant death;

- Then two groups of 30 medical students (6.) tested the double scenario prototype and parameterized it;

- The final application was then tested by 35 educators of 10 kindergarten sites in Lausanne (Switzerland), who completed an anonymous questionnaire;

- Regrouped results were analyzed by (1.) and (2.).

\section{A. User-centered design}

Patient care and monitoring can be considerably improved over time with the use of technological health devices [21]. That's why information and communication technologies like serious games have become an integral part of the provision of health care: a broad range of new electronic-health ( $e$ Health) applications has emerged [...]. All these applications promise patients, nurses, doctors, and administrators to efficiently access relevant health information, enhance patient care quality, reduce healthcare errors, increase collaboration, and encourage the adoption of healthy behaviours [23].

Those two situations already lead to a software requiring a strong development flexibility and pushed towards the development of a point-and-click game, allowing: (1) the management of possible actions depending on previous conditions, (2) the management of items, and (3) a system where the team can quickly add new content or update it through editable files.
When managing vital emergencies, we identify stress factors such as inexperience with first aid gestures (ability to identify the situation and delegate, effective leadership, lack of mastery of techniques, organizational problem, etc.) fear of error and forgetfulness, reflection and rapid decision-making as well as the projection of the idea of death. SG4ER aims to make the user aware of stressful situations and help him implement strategies to evolve such as improving time and organization management, self-confidence development and the best control of resuscitation techniques and equipment. To transcribe as best as possible the feeling of emergency in the game, we chose to let the user explore the environment by giving minimal information. He must become aware of the important knowledge he requires when confronted to an urgent situation, such as the location of the pharmacy, the important items he must gather or being able to take upon useless actions.

Furthermore, each action takes actual time and an useless action has an added negative effect to the solving of the situation. Finally, the call to the emergency services rises questions that have a limited time to be answered, giving a final touch to the stress related to giving the vital information as fast as possible.

\section{B. Engineering model}

As shown in the previous section, this project has been developed with user-centered methodologies (AGILE, Scrum [18]). This methodology has been used as a fastiterative process to develop a minimum viable prototype integrating all the game mechanics clinical experts needed to convey educative content. Scrum allowed quick shifts in direction based on multiple proof-of-concept iterations and ensure the product felt as stressful to play as a real situation.

The app goal was to reach as many people as possible and thus ensure a large compatibility with various machines. Qt enabled us to develop without constrains for recent MacOS versions and Windows XP, 7, 8 and 10. Furthermore, a Linux version could also be released. To be able to run the application, the user must download the installer, run it and then run the game. The time needed to finish both available levels amounts to about 20 playtime minutes.

\section{External files}

The game is mainly composed of images taken from an actual child care center. Some pictures are meant to navigate within the corridors and some represent scenes where the user will be able to do actions. The application also uses icons to help illustrate user options and sounds to make his actions more immersive. The sounds can range from bandaging noises to a baby crying. Some of the music is also used to set up the scene mood and to deepen the experience. The other important files are the level descriptors containing all the scene configurations, options and questions. Those files put into relation the different data of a level and enable a flexible creation and edition of levels. The scoring files, run on a separate file to facilitate editing, are together with the level 
descriptions and they are used to compute the user's final score and generate a feedback to user's different actions.

\section{User profile and email feedback}

At each start of the app, the user is asked to input his email which is then used to receive a PDF file of his session results with his different scores and feedback information. There currently is no system saving the user profile between launches. A short resume of the results is sent both to a centralized email for data collection and to the email the user inputs when starting his session. No email validation is done, and a fake email can be used to access the program. This system is the demonstration of the potential feedback and monitoring system that could later be implemented.

\section{E. The game}

The two levels place themselves in a child care institution. The user is driven through four different phases. The first one consists of a contextualization of the level and important data about the center location and access which include: a map of the scene, the street name, the city, the floor and entry code of the building. The user must remember this information and can look it up during his play-through. Once the user is ready, he is put at the beginning of the scene and must explore it to find what the trouble is. He can look through medical files, pick up items and realize various actions to analyze and solve the situation.

At one point, the user will have to call the emergency center that will ask him timed questions. The time limit is there to give the emergency feeling usually given by call receptionists. In this part, his possible answers depend on his actions in the scene. Finally, the session ends as the player receives, by email for privacy and back-up, his score and advice for further improvement as a feedback. She or he will also be able to retry the level or go back to the level selection.

One of the most critical design decisions made for the game was tightly linked to the feeling of urgency one has in stressful situations. Therefore, the user has access to a relatively large list of futile or even negative actions to make him feel the lack of external guidance you could have in those situations. Furthermore, the game never gives signs if an action is correct of wrong during the procedure and never lets through if the care is actually properly finished.

\section{F. Score analysis}

A user's play-through will amount to numerous actions evaluated between correctness and timeliness. Each action will impact the total level time as well as the speed at which the user takes care of the patient. Therefore, all the user's actions during the session, as well as his choices during the call to the emergency services, are analyzed by a dedicated scoring module. The output consists of an action score, a time score, a call score and his two best and worst actions.

The Fig. 5 summarizes the different parts of the application and their communication with each other. Each number can be summarized as follows:

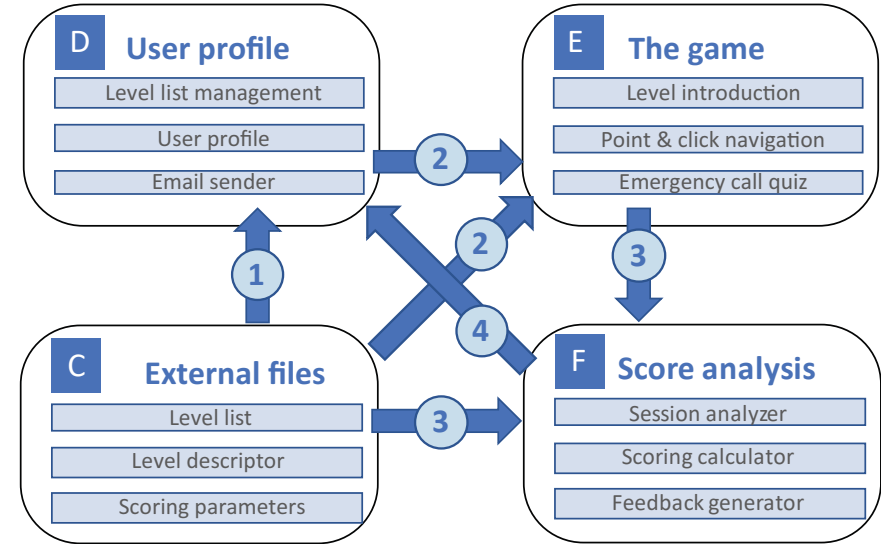

Fig. 5. Presentation of the application core parts and their communication with each other.

1) Loading of the external files describing the available levels and opening of the main menu;

2) The user selected the level and loaded the required images, level descriptions and sounds to render the level mood;

3) Level completion, score analysis file load and scoring computation;

4) Email containing user's score and feedback + level selection screen again.

The Fig. 6 presents the 10 steps of the tutorial introducing the game structure: (1) main menu; (2) mini map; (3) interface: phase I main view, reaction to emergency situation; (4) description of the three left info window; (5) explanation on why each action will cost time and why choice is therefore crucial; (6) Navigation; (7) how to select and take objects, here in the pharmacy kit; (8) interface: phase II beginning, the emergency call; (9) procedure of official questions asked; (10) feedback with score on amount of time spent, correct/wrong actions, and correct/wrong answers to emergency service.

A relatively simple tutorial was implemented at this stage regarding basic information and a detailed map is given to the user before starting the level. Nonetheless, the current feel of the game forces the user to actually explore, deduct and memorize where important material is stored in the levels facilities.

\section{EXPERIMENTAL APPROACH}

From an educational point of view, this proposal is a simulation game based on a social constructivist model. The user, thanks to his experience, will understand the factors involved and the interrelations between them, with an understanding more than a simple absorption. He becomes the actor of his apprenticeship: simulations advocate learning by discovery and action -i.e. learning by doing [4](page 7).

Applying his experience in different scenarios, the user gains control of his own skills. Indeed, the goal, under these circumstances, is the completion of a mission associated with the role assigned to it in the scenario. In addition, this 

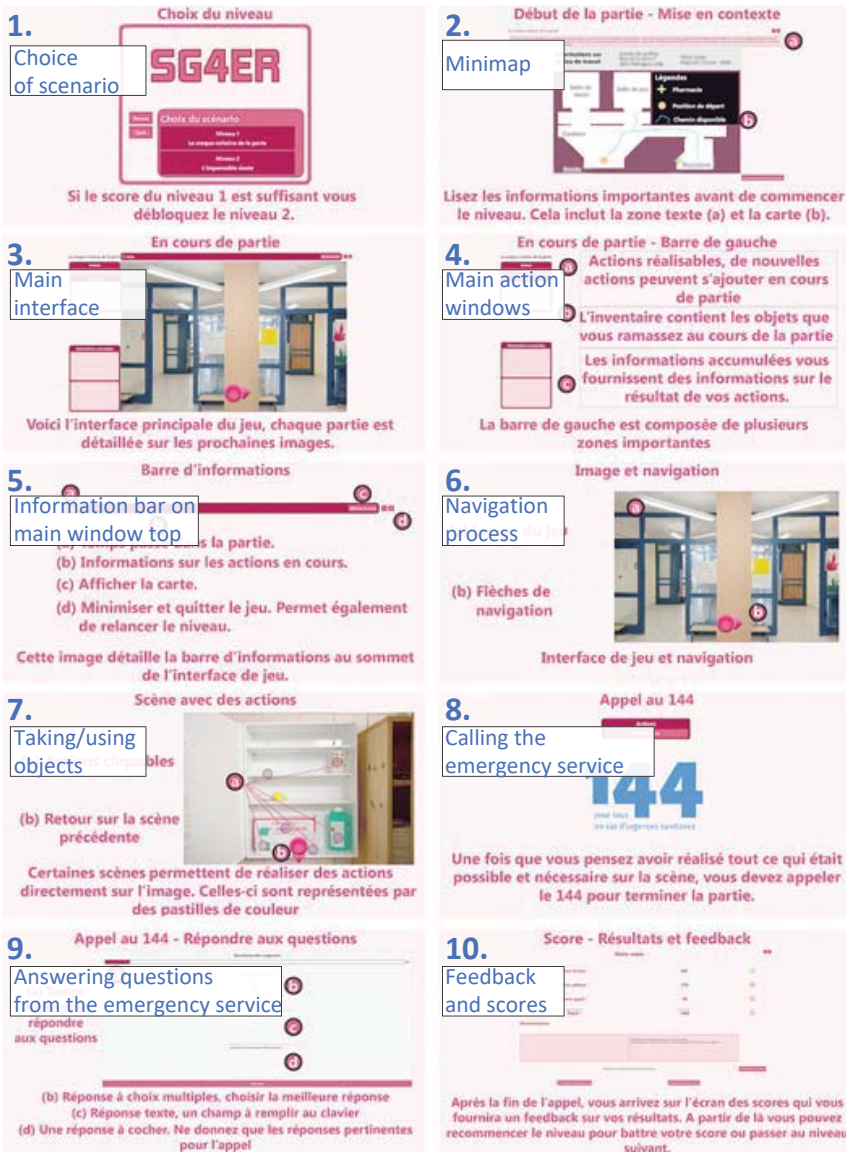

Main action actions peuvent s'ajouter en cours windows dinventaire contient les objets que

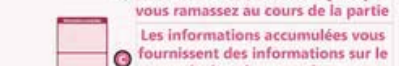
Lournissent des informations sur
résultat de vos actions. La barre de gauche est composée de plusieurs

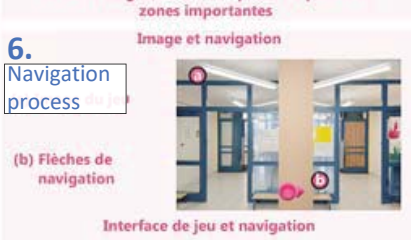

6. ge et navigatio

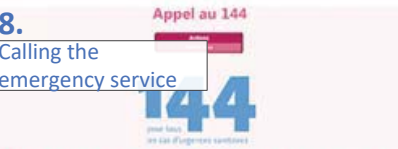

Une fois que vous pensez avoir reallisé tout ce qui était possible et nécessaire sur la scène, vous devez appeler

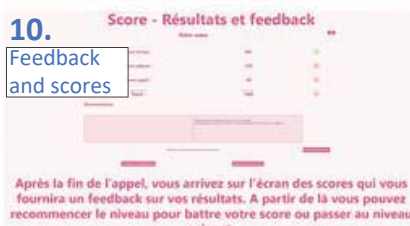

Fig. 6. Presentation of the tutorial guide in 10 steps: from main menu (1.) to user feedback and scoring (10.).

resolution process will allow him to develop new skills such as leadership and/or social, communication and interpersonal skills. It is also possible to introduce the pleasure dimension in the situations developed. This pleasure is the opportunity that the game gives to excel, to confront challenges while having the feeling to control the situation, but also to interact with others to collaborate. Thus, this approach offers a privileged way to involve the users, in particular by immersing in a realistic universe and motivational springs such as the transition to the next level. The desired impact is that the players appropriate the problems designed for learning, in action. In this sense, serious play is an experiential learning approach.

\section{Results}

\section{A. Platform presentation}

Even if only two scenarios were completed, the 524 pictures (with 321 actually cleaned and used) of this SG were taken in a single day. Samples of them are presented in Fig. 7. Their final resolution input into the game is of 1920 by 1080 pixels. When facing the images of the case user had to handle, the result was surprisingly immersive considering their relatively strong reactions. The final app remains a proof of concept helping people that were never confronted to child care and an effective tool for knowledge assessment. This fact demonstrates that even a low-cost development tool can have a strong impact allowing positive actions to be taken upon being confronted to actual emergency cases.

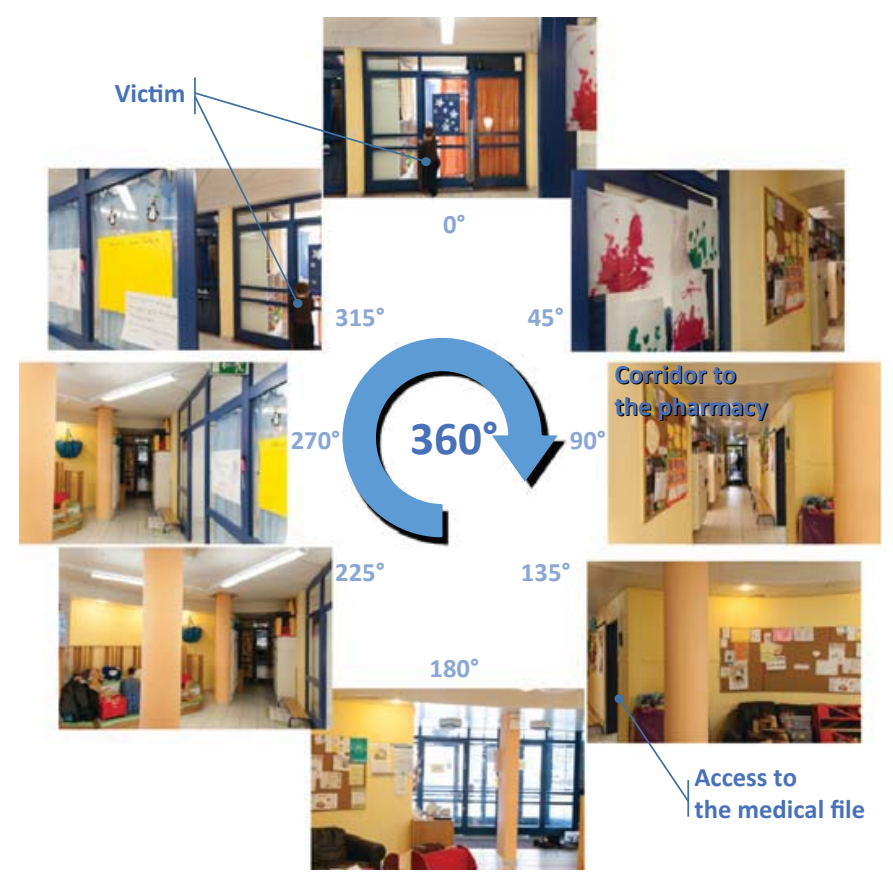

Fig. 7. This figure illustrates the picture taken to be able to rotate over 360 within the game.

Images of scenarios 1 and 2 are illustrated by Fig. 8 with three sets of pictures (among 321 selected):

- Top set: these images are used in both scenarios since they represent the environment where the player looks for (1) information about the child (e.g. checking for allergies or special medical conditions) and (2) important basic medical supplies in the pharmacy (e.g. gloves);

- Middle set: four major steps from the first scenario with (1 and 2), the discovery of the four-year-old child with his fingers stuck in and half-sheared by the metallic door and (3 and 4), preparation and bandage result;

- Bottom set: four possible events from the second scenario once the baby is found unconscious in his bed (i.e. at the beginning, the player does not know it is due to a cardiac arrest). (1) taking the temperature; (2) using manual breathing system; (3) checking for reaction; (4) applying a manual cardiac massage.

\section{B. Survey results}

The analysis of the results aimed at evaluating both scenarios and categorizing the carried-out raw data with regard to the different items of the five step basic questionnaire: (1) Game relevance (quality and realism of the scenario-possible learning); (2) Graphics (image quality); (3) Ergonomics / navigation-handling; (4) Quality of the proposed answers; and (5) Tutorial. 


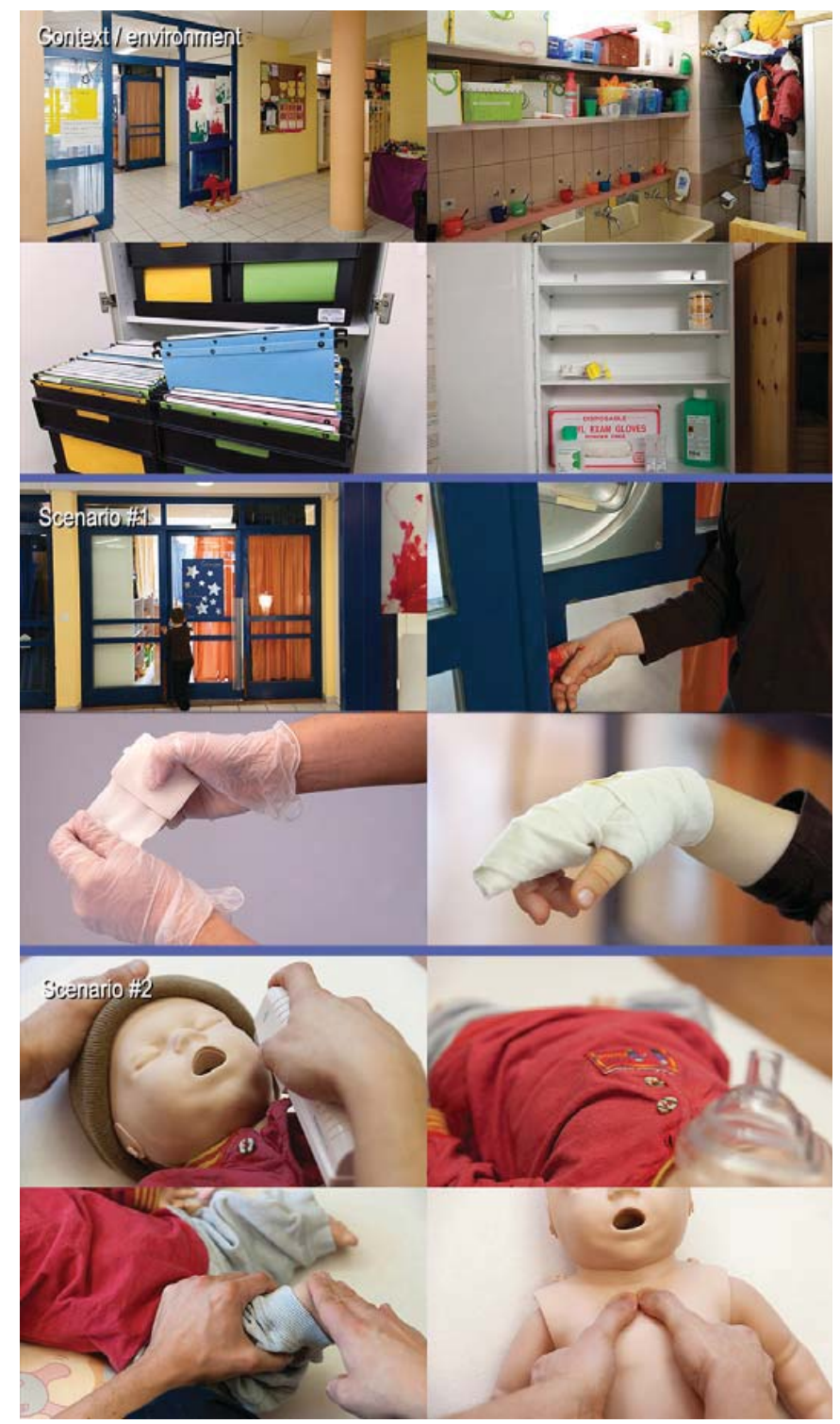

Fig. 8. Three sets of pictures from both scenarios: Environment and seeking for basic child information or medical supplies (top); first scenario called "Deep double-finger wound" (middle); second scenario "Cardiac arrest in infants" (bottom).

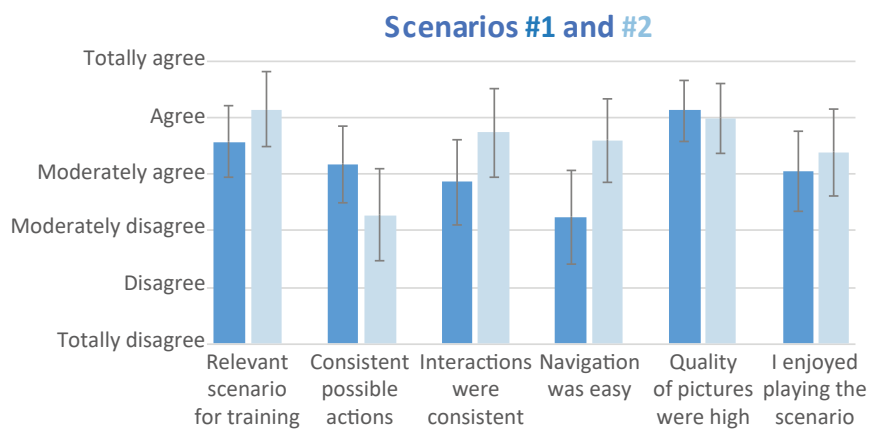

Fig. 9. This figure illustrates how users reacted to both scenarios.
The first conclusions is that the SG has been validated as an original learning tool since it is has an impact and its approach is relatively original. It certainly needs to be improved, especially in terms of navigation ease, graphic quality (adding pictures), and several more levels - at least three we believe. Even if photographs are by nature realistic, it remains the issue of information readability. We notice that the second scenario was more appreciated by end-users just by the facts that navigation was easier and a richer scenario.

Educators expressed a greater enthusiasm using this tool and gave complete and accurate comments. Their need for continuous training is probably a possible explanation. Nonetheless, a significant part of them clearly showed some difficulty handling this type of tool just because they are not video game players. On the contrary, Bachelor students did not have any problem using this tool even though they had comments on the navigation and the tutorial; they were sensitized during their training to work in simulation (intelligent dummy, role play and so on) and are part of the generation of digital natives.

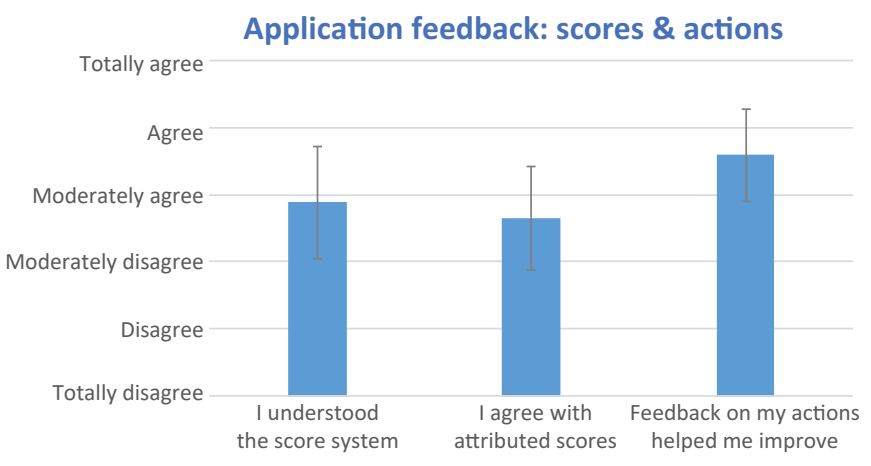

Fig. 10. This figure illustrates how users reacted to both scenarios.

Our premise is that acquiring new knowledge in a pleasant way, by applying the principles of gamification to the emergency care process, can only improves with enthusiasm its acquisition as well as commitment.

\section{Discussion}

This project has demonstrated that it is possible to enrich and increase first-aid education through the introduction of information and communication technologies by developing a low cost device.

Skill acquisition can be challenging, and this educational tool can be identified as a new pedagogical approach and a learning tool. Nevertheless, the scenario must as much as possible reflect situations that are encountered to properly train the emergency response and mobilize the user. We believe, this prototype will have to be remodelled to improve the readability of possible actions, which will have to gain in precision and variety. It is an exercise that should conclude with indications to develop knowledge and a debriefing with a health professional. 
A lot of potential developments remain - Users have considered the game as a efficient time for learning and reflection allowing a confrontation with the reality of care situations. This experience lets us imagine the development potential of games around any serious health topic, combining pleasure and learning. Furthermore, direcly based from user comments, here follows a non-exhaustive list in terms of software improvements:

- First of all, translating the app from French to English

- Adding the three levels, already analysed and selected by social experts;

- Adding spherical views;

- Using web-based technologies or converting into a tablet/mobile app game;

- Using node-based frameworks to create richer contents with conditional dialogues, higher choice impacts, and simpler editors for health partners;

- Increasing content diversity, especially with wrong possible actions;

- Redesigning UX and improving the spatial setting; a Google Streetview-like system would strongly help the user not to lose himself;

- Using gamification principles integrated within the work ecosystem so as to increase adoption;

- Adding multiplayer mode to improve the link to real life situations where education professionals work collaboratively [16].

Finally, enriching the game with a legal dimension would allow to answer questions such as: What actions are or not authorized or expected? What is the commitment of the responsibility of professionals in this type of situation? This useful perspective, however, implies the SG to be adapted to legal frameworks of each structure, each municipality, and each country - bringing a relatively large amount of work.

\section{ACKNOWLEDGMENT}

This work was supported by the HE-Arc (HES-SO) internal funding system, grant entitled "SG4ER" (2015-2018). The authors would like to thank all engineering and health department students for their help, testing, and contribution during internship, configuration, and user-tests phases. Special thanks are also due to Mrs Gutierrez Montserrat for the precious English review of the manuscript and to Mrs. Valentine Brodard for all the photographic shots. Finally, we would like to thank the Children's Day Care Service, its management, its directors and in particular Mrs Ana Lattion and Mrs Nathalie Bigot as well as the educator Mrs Françoise Pellein for their active participation in scenarios inspired by real facts.

\section{REFERENCES}

[1] Babu, S. K., McLain, M. L., Bijlani, K.,as well as the educator Mrs Franoise Pellein, "Collaborative Game Based Learning of Post-Disaster Management: Serious Game on Incident Management Frameworks for Post Disaster Management”. IEEE Eighth International Conference on Technology for Education (T4E), 2016.
[2] Baranowski, T., Blumberg, F., Buday, R., et al., "Games for Health Journal.Feb 2016, Games for Health for ChildrenCurrent Status and Needed Research", Institute of Digital Media and Child Development Working Group on Games for Health, vol.4, nu.6, 2015.

[3] Chan, W-Y., Qin, J., Chui, Y-P., and Heng P-A., "A Serious Game for Learning Ultrasound-Guided Needle Placement Skills". IEEE Transactions on Information Technology in Biomedicine, vol.16, nu.6, pp.10321042. 2012.

[4] De Vries, E., "Les logiciels d'apprentissage : panoplie ou éventail ?", Revue Française de Pédagogie, vol.137, pp.105-116, 2001.

[5] Diehl, L., Souza, R., Alves, J., et al., "a Serious Game to Teach Insulin Therapy to Primary Care Physicians: Design of the Game and a Randomized Controlled Trial for Educational Validation", JMIR Res Protoc; vol.2, nu.1, 2013.

[6] Gobron, S., Zannini, N., Wenk, N., et al., "Serious games for rehabilitation using head-mounted display and haptic devices", book chapter of Augmented and Virtual Reality, Vol.9254, Lecture Notes in Computer Science, pp.199-219, 2015.

[7] Knight, J., "Serious gaming technology in major incident triage training: A pragmatic controlled trial", Resuscitation journal, vol.81, nu.9, pp.1175-1179, September, 2010.

[8] Li, K., Hall, M., Bermell-Garcia, P., "Measuring the learning effectiveness of serious gaming for training of complex manufacturing tasks". Simulation and Gaming, vol.48, nu.6, pp.770-790, December 1, 2017.

[9] Matsunaga, R., de Oliveira Moraes, R., Borges, M., et al., "Development of a serious game for children with hemophilia", IEEE 3rd International Conference on Serious Games and Applications for Health (SeGAH), pp.1-6, 2014

[10] Mikuszewski, R., Szczesna, A., Grudzinski, J., et al., "The psychology serious game prototype for preschool children", IEEE 1st International Conference on Serious Games and Applications for Health (SeGAH), Braga, 2011, pp. 1-4, 2011.

[11] Peden, M., Oyegbite, K., Ozanne-Smith, J., et al., "Rapport mondial sur la prévention des traumatismes chez l'enfant.”, p.44, 2008.

[12] Peden, M., Oyegbite, K., Ozanne-Smith, J., et al., "World report on child injury prevention", Geneva, World Health Organization, p.44, 2008.

[13] Peden, M., Oyegbite, K., Ozanne-Smith, J., et al., "Recommandations pour laccueil collectif de jour des enfants". Department of Training, Youth and Culture, Youth Protection Service, Lausanne, 2010.

[14] Qin, J., Chui, Y-P., Pang, W-M., et al., "Learning Blood Management in Orthopedic Surgery through Gameplay". IEEE Computer Graphics and App., vol.30, pp.4557, 2010.

[15] Rea, T. and Page R. "Community Approaches to Improve Resuscitation After Out-of-Hospital Sudden Cardiac Arrest", the Resuscitation to recovery journal, Circulation, vol.121, pp.11341140, 2010.

[16] Ricciardi, F. and Tommaso De Paolis, L., "A Comprehensive Review of Serious Games in Health Professions", International Journal of Computer Games Technology, Article ID 787968, 2014.

[17] Richard L. Page, MD., "Community Approaches to Improve Resuscitation After Out-of-Hospital Sudden Cardiac Arrest”. Circulation, vol.121, pp.1134-1140, 2010.

[18] Schwaber K. andBeedle M., "Agile Software Development With Scrum", prentice hall, October 21, 2001

[19] Sisto, M., Zare, M., Ouerhani, N., Bolinhas, C., et al., "Virtual Reality Serious Game for Musculoskeletal Disorder prevention", Augmented and Virtual Reality: 5th International Conference (AVR 2017), Springer, Ugento, Italy, June 12-15, 2018.

[20] Tanaka, Y. Taniguchi, J., Wato, Y., et al., "The continuous quality improvement project for telephone-assisted instruction of cardiopulmonary resuscitation increased the incidence of bystander CPR and improved the outcomes of out-of-hospital cardiac arrests". The Resuscitation journal, vol.83, nu.10, October, pp.1235-1241, 2012.

[21] Wattanasoontorn V., Boada i., Garca r., and Sbert m., "Serious games for health", entertainment computing, 4, pp.231247, 2013.

[22] WISQARS institutional report, "Unintentional fatal and nonfatal injuries, children ages 19 and under". Centers for Disease Control and Prevention, National Center for Injury Prevention and Control. Web-based Injury Statistics Query and Reporting System (WISQARS), 2018.

[23] Yeh, K. H., Lo, N. W., Wu, T. C., and Wang, C., "Secure e-health system on passive RFID: Outpatient clinic and emergency care". International Journal of Distributed Sensor Networks, Vol.2013, 2013.

[24] Zipes DP., Wellens HJJ., "Sudden cardiac death". Circulation, vol.98, pp.2334-51. Thomas D. Rea, MD, MPH, 1998. 